\title{
A Stochastic Model for Volumetric Errors
}

Rosenda A. Valdés arvaldes@ig.com.br

Denise P. V. Sato denise@4all.com.br

Roxana M. Martinez Orrego rox.maria@gmail.com Aeroalcool Tecnologia Ltda. 14403-450 Franca, SP, Brazil
The present work aims to determine mathematical equations that describe the behavior of the components of the volumetric error in Three Coordinates Measuring Machines (3CMM). A general methodology using techniques of multiple regressions is presented. Such methodology, applied to a moving bridge type 3CMM, has made it possible to obtain, in a simple way, three regression equations from data collected through a direct calibration procedure, more specifically, the space grid method. The proposed model was statistically and experimentally evaluated. The statistic evaluation covers the calculation of the correlation coefficient of the samples, a residue analysis and the hypothesis verification. The experimental evaluation was made through the comparison of results expected by the model with the results obtained from the measurements of a ball bar. From these results, it is possible to verify that the model is adequate and that it is good in predicting volumetric errors in the machine.

Keywords: Space grid method, ball bar, multiple regression, Abbè offsets and least squares

\section{Introduction}

The contemporary times, characterized by great scientific discoveries, an accelerated technological development and global economy, has also brought the evolution of productive processes. The global character of the commercial relations, the competition, and the struggle for bigger slices of the market made companies to invest in the search of new technology with the objective of increasing the productivity and the quality of products. As a consequence, modern tooling equipment has been incorporated to the industrialized world where the products are manufactured with lower tolerances and in larger quantities. Therefore, it has become necessary integrate to these systems with faster and more precise, more flexible and more reliable means of control.

The Three Coordinates Measuring Machines are apparently the devices that supply these needs, representing one of the most advanced equipment used in modern metrology. It may be said that these machines present simplicity of operation, flexibility, accuracy and also, it permits to take fast and precise measurements of complex structures as well as simultaneous control of several metrological characteristics of a piece (Kunzmann \& Waldele, 1988).

However, the performance of $3 \mathrm{CMM}$ is limited due to the presence of Abbè offsets, the difficulty of assembling in three axes, theoretically orthogonal, and also due to imperfections caused by the tooling processes that take place in several mechanical elements which compose the system. These factors act together, combining to each other in a complex way all over the volume of the machine, contributing to the so called volumetric errors. Every reading, which is a result of a measurement, will always be subjected to errors and therefore it is necessary to develop methods so that these errors are minimized.

In this sense, the objective of this work is to formulate the components of the volumetric error of a 3CMM of a moving bridge type using techniques of multiple regression aiming the prediction of the volumetric error in any given point of the working volume of the machine.

\section{Nomenclature}

$$
\begin{aligned}
& C_{S q}=\text { correction due to error of the mechanical square. } \\
& L_{L V D T}=\text { reading taken by LVDT. } \\
& R_{L V D T}=\text { LVDT resolution. }
\end{aligned}
$$

Paper accepted: May, 2005. Technical Editor: Anselmo E. Diniz.
$D=$ measured displacement.

$D_{1}$ and $D_{2}=$ diameters of balls 1 and 2, respectively.

$D_{B M}=$ measured length of the bar.

$D B_{M x}, D B_{M y}$ and $D B_{M z}=$ projections of the length of the bar in the preferential directions.

$D_{B P}=$ standard length of the bar.

$D_{B R}=$ nominal length of the bar.

$E_{B x}, E_{B y}$ and $E_{B z}=$ projections of the $E x, E y$ and $E z$ in the direction of the bar, respectively.

$E P o s=$ value indicated by laser.

$E v=$ volumetric error.

$E x, E y$ and $E z=$ components of volumetric error.

$M=$ value indicated by machine.

$P=$ reference value (Laser).

$P_{E B}=$ projection of the volumetric error in the direction of the bar.

$r^{2}=$ correlation coefficient

$R_{\text {Laser }}=$ laser resolution

$R_{M M 3 C}=$ machine resolution

$v_{\text {eff }}=$ effective degree of freedom

$X, Y$ and $Z=$ coordinates

$X_{0}, Y_{0}$ and $Z_{0}=$ coordinates of center of ball.

$X^{2}, Y^{2}, Z^{2}, X Y, X Z$ and $Y Z=$ variables of regression.

Greek Symbols

$\alpha_{P}=$ coefficient of thermal expansion of the laser beam.

$\alpha_{E}=$ coefficient of thermal expansion of the scale (glass).

$\alpha_{S q}=$ coefficient of thermal expansion of the mechanical square.

$\alpha, \beta$ and $\gamma=$ angles which define the position of the bar with the directions $O X, O Y$ and $O Z$, respectively.

$\Delta T=$ difference between the room temperature and the reference temperature

$\beta_{i}=$ least squares estimators

$\delta T=$ difference between the scale temperature and the reference temperature

$\delta x(x), \delta y(y)$ and $\delta z(z)=$ positioning error at axis $X, Y$ and $Z$, respectively.

$\delta x(y)$ and $\delta Z(y)=$ straightness error of axis $Y$ direction $X$ and $Z$, respectively.

$\delta x(z)$ and $\delta y(z)=$ straightness error of axis $Z$ direction $X$ and $Y$, respectively.

$\delta y(x)$ and $\delta z(x)=$ straightness error of axis $X$ direction $Y$ and $Z$, respectively.

$\delta \theta x(x)$ and $\delta \theta y(y)=$ angular error Roll to axis $X$ and $Y$, respectively. 
$\delta \theta x_{0}, \delta \theta y_{0}$ and $\delta \theta z_{0}=$ orthogonality errors.

$\delta \theta y(x), \quad \delta \theta x(y)$ and $\delta \theta x(z)=$ angular error Pitch to axis $X, Y$ and $Z$, respectively.

$\delta \theta z(x), \delta \theta z(y)$ and $\delta \theta y(z)=$ angular error Yaw to axis $X, Y$ and $Z$, respectively.

$\Delta T_{E}=$ difference between the mechanical square temperature and the reference temperature.

$\varepsilon_{i}=$ residues of regression.

\section{Subscripts and Superscripts}

$i=$ points where volumetric error components were collected.

\section{Technical Characteristics of the Measurement System}

The structure of the $3 \mathrm{CMM}$ serves as a support and permits the movement of a sensor in three orthogonal axes $X, Y$ and $Z$ of 457, $610,381 \mathrm{~mm}$ in length, respectively. These dimensions are denominated work capacity.

The coordinates $\mathrm{X}, \mathrm{Y}$ and $\mathrm{Z}$ determine the position of the points on the surface of the pieces in space. The $3 \mathrm{CMM}$ was intentionally designed to measure these values. However, it is impossible to obtain the true or real coordinates of the points due to many factors that interfere in the process of measurements.

In order to determine the relation input - output of the system "measuring machine" it is necessary to define and classify the variables which are involved in the measuring process, and for that, it is necessary to carry out a preliminary analysis of these variables.

From this analysis it is noted that the coordinates of the points may be considered as the input to the system or preliminary inputs. Each one of these coordinates may be influenced by many of the 21 geometrical errors that affect the result of a measurement. These geometrical errors constitute what may be called interfering inputs to the system. Also, it must be considered the modifying inputs, in this case, temperature, humidity and vibrations are kept under control and their influences were neglected.

The combination of the geometrical errors in a point in space in each one of the preferential directions is named component of the volumetric error. These three components are considered outputs of the system. It is then defined the inputs and outputs of the measuring machine system. Fig. 1.

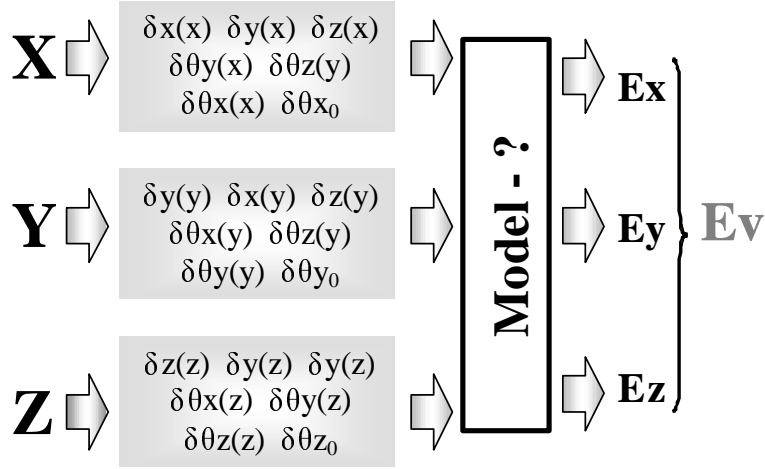

Figure 1. Representation of the ЗСМM measuring system.

Note that for the determination of the relation input-output the measuring machine system may be considered as being three subsystems. Each one of them has three inputs, which are the coordinates of the measuring points $\mathrm{X}, \mathrm{Y}$ and $\mathrm{Z}$, and only one output, which is the component of the corresponding volumetric error $E x, E y$ or $E z$ according to the case.

Once defined the inputs and outputs of the system it is possible to classify it. According to the control theory presented in Ogata (1982) and Harris (1996), the system 3CMM of Moving Bridge type may be classified as:
Invariable in time: The value of the volumetric error in any instant in time depends only on the values of the coordinates $X, Y$ and $Z$ and not on the moment in time in which the coordinates are measured.

According to the position: the volumetric error is a continual mathematical function of three variables, in that case, the coordinates $X, Y$ and $Z$ which define the position of the measured points.

Quasi-static: the value of the volumetric error in any position depends only of the current position and not on the previous or future positions. The thermal memory of the moving bridge $3 \mathrm{CMM}$ system can be considered null if temperature is controlled.

The system may be classified as MISO: the system presents multiple inputs and single output. According to the above classification, the relation between input and output of the measuring machine system may be expressed through a mathematical model which describes the components of the volumetric error as a function of the position $X, Y$ and $Z$, represented in the system of Eq. (1).

$$
\begin{aligned}
& E x=f_{1}(X, Y, Z) \\
& E y=f_{2}(X, Y, Z) \\
& E z=f_{3}(X, Y, Z)
\end{aligned}
$$

\section{Mathematical Equation.}

Regression techniques allow expressing the existing relation between a dependent or response variable and one or more independent or input variables (Box et al, 1978; Draper \& Smith, 1981 and Hoffmann \& Vieira, 1973). Such techniques can be applied to sampled data in order to estimate the value of an unknown variable from one or more variables whose value is known (Spiegel, 1974).

To determine the existing relation among the variables involved in a measurement process was use the regression techniques. Were, the coordinates of the points $X, Y$ and $Z$ and the components of the volumetric error $E x, E y$ and $E z$, represented the inputs and outputs of the measuring machine system.

Was proposed a multiple linear regression equation for each of the coordinated axis, for the axis $X$ we have:

$$
E_{X i}=\beta_{X 0}+\beta_{X 1} X_{i}+\beta_{X 2} Y_{i}+\beta_{X 3} Z_{i}+\varepsilon_{X i}
$$

Where $E_{X i}$ represents the component of the volumetric error in the direction $X$ for the different positions; $\beta_{X_{0}}, \beta_{X_{1}}, \beta_{X_{2}}$ and $\beta_{X_{3}}$ are the regression coefficients; $X_{i}, Y_{i}$ and $Z_{i}$ are the coordinates of point $i$; an $i=1,2, \ldots, n$ and $\varepsilon_{X i}$ are the residues of the regression of the regression.

The Eq. (2) is called multiple linear regression equation for having multiple independent variables. The estimators of minimum square are determined in such a way that the sum of the squares of the residue are minimized, i.e.

$$
\frac{d\left(\sum_{i=1}^{n} \varepsilon_{x_{i}}^{2}\right)}{d x_{i}}=\frac{d\left(S\left(\beta_{X 0}, \beta_{X 1}, \beta_{X 2}, \beta_{X 3}\right)\right)}{d x_{i}}=\frac{d\left(\sum_{i=1}^{n}\left(E_{X i}-\beta_{X 0}-\beta_{X 1} X_{i}-\beta_{X 2} Y_{i}-\beta_{X 3} Z_{i}\right)^{2}\right)}{d x}=0
$$

in this case it is convenient to write the multiple regression in a matrix form, Eq. (4).

$$
E_{X}=H \beta+\varepsilon_{X}
$$


where

$$
H=\left[\begin{array}{cccc}
1 & X_{1} & Y_{1} & Z_{1} \\
1 & X_{2} & Y_{2} & Z_{2} \\
\vdots & \vdots & \vdots & \vdots \\
1 & X_{n} & Y_{n} & Z_{n}
\end{array}\right], \quad E_{x}=\left[\begin{array}{l}
E_{X 1} \\
E_{X 2} \\
\vdots \\
E_{X n}
\end{array}\right], \quad \beta=\left[\begin{array}{l}
\beta_{X 0} \\
\beta_{X 1} \\
\beta_{X 2} \\
\beta_{X 3}
\end{array}\right] \text { e } \varepsilon_{X i}=\left[\begin{array}{l}
\varepsilon_{X 1} \\
\varepsilon_{X 2} \\
\vdots \\
\varepsilon_{X n}
\end{array}\right]
$$

The estimates of the regression coefficient in Eq. (4) may be determined using the least squares method. For that it is necessary that $\left(H^{T} H\right)$ be an invertible matrix, Eq. (5).

$$
\hat{\beta}_{X}=\left(H^{T} H\right)^{-1} H^{T} E_{X}
$$

Once the numerical values of the regression coefficient are known, it is then possible to determine the predicted values of the component of the volumetric error in the direction of the axis $X$ and the residue, using the Eq. (6) and (7), respectively.

$$
\begin{gathered}
\hat{E}_{X}=H \hat{\beta}_{X} \\
\hat{\varepsilon}_{X}=E_{X}-\hat{E}_{X}=E_{X}-H \hat{\beta}_{X}
\end{gathered}
$$

In a similar way, regression equations were proposed to equation the components of the volumetric error in the directions $Y$ and $Z$.

$$
\begin{gathered}
E_{X i}=\beta_{X 0}+\beta_{X 1} X_{i}+\beta_{X 2} Y_{i}+\beta_{X 3} Z_{i}+\varepsilon_{X i} \\
E_{Y i}=\beta_{Y 0}+\beta_{Y 1} X_{i}+\beta_{Y 2} Y_{i}+\beta_{Y 3} Z_{i}+\varepsilon_{Y i} \\
E_{Z i}=\beta_{Z 0}+\beta_{Z 1} X_{i}+\beta_{Z 2} Y_{i}+\beta_{Z 3} Z_{i}+\varepsilon_{Z i}
\end{gathered}
$$

\section{Obtaining the Components of the Volumetric Error in the 3CMM}

The direct calibration of the measuring machine using the space grid method consists of the direct measurement of each component $E x, E y$ and $E z$ of the volumetric error. As shown in Fig. 2, the machine volume is divided into small volumes or cubes, by the generators or measurement lines, which are parallel to each one of the three preferential directions.

Position measurements are realized along the defined generators (Burdekin et al, 1984), whose results comply a combination of all effects of Abbè offsets and all uncertainties that have influence in one given direction. From the results obtained on these measurements, it is possible to calculate the volumetric error directly, without the need of modeling the machine structure (Martinez Orrego, 1999).

Initially, an analysis of the machine was carried out in order to define the point where the coordinated system reference would be placed. The point $(0,0,-260 \mathrm{~mm})$ was chosen in relation to the zero of the machine. The volume to be modeled was divided by straight lines parallel to each one of the axes of the machine, forming a net with a total of 147 generators. The measuring positions were defined as the intersection points of the generators.

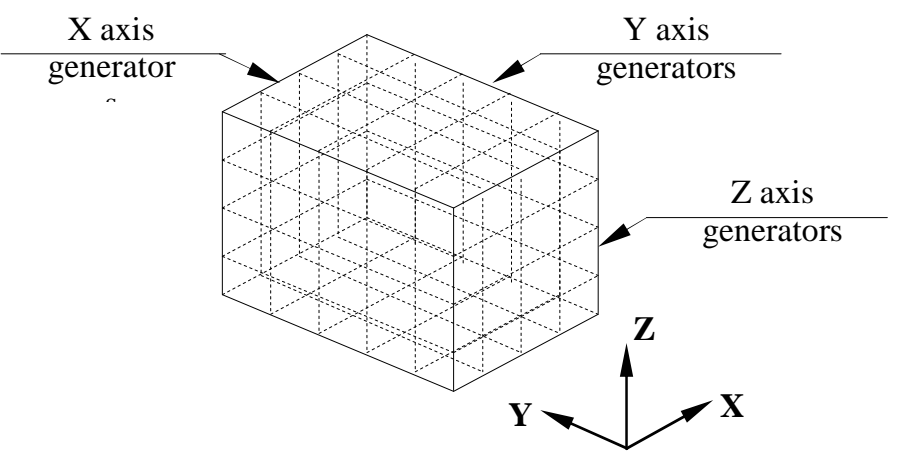

Figure 2. Volumetric calibration with space grid method.

Each component of volumetric error can be measured in two different planes, however in order to save time, they have been measured in one plane only. Therefore, the components $E x, E y$ and $E z$ have been obtained in the planes $X Z, Y Z$ and $X Y$, respectively, using a laser interferometric system of Hewlett Packard, model HP5528A. Some computational algorithms were employed to accomplish data acquisition and to establish communication among a computer, the interferometric system and the machine scales.

The position error is calculated as the difference between the value indicated by the machine and the value indicated by the laser, Eq. (9).

$$
E_{i i}=\text { value indicate by machine }- \text { value indicate by laser }
$$

The readings were taken at every $25 \mathrm{~mm}$ in the measuring range of each axis of the machine. For each generator, five complete measuring cycles were accomplished, each one consisting of five forward and five backward measurements. It is known that due to the influence of the errors in the shape and position and in the lack of orthogonality between axes, the value of the error in the initial point of the generator in a given direction is not equal to zero. In these points, the errors take the value zero due to an imposition of the test, once the interferometer must be reset before the beginning of each measurement procedure. Therefore, in order to obtain the real values of the errors it is necessary to make a correction of the initial points of each one of the generators. For that, a direct measure of the correction factors for each measuring plan was carried out. More information can be found in Valdés (1999).

During the measurement procedure a mechanical square aligned to the axis of the errors that are to be corrected and one LVDT type transducer placed on the test point was used (Fig. 3).



Figure3. Square standard and LVDT probe placement.

The correction values are the values taken in the point where the generator goes through the square. These correction factors constitute a sum of the straightness, angular and orthogonal errors. Each measure was repeated five times, in order to obtain a group of 
values that permit to correct the curves of the volumetric component error $(E x)$. The correction factors of the zeros were measured in a similar way and the curves of errors $E y$ and $E z$ were corrected.

\section{Obtaining the Model}

Once all the results of the measurements of the components of the volumetric error have been obtained, they were substituted in the system of Eq. (8) and introduced in a computer program with the purpose of determining the coefficients of the regression equations. The three equations that were found had relatively high correlation coefficients and the residue presented good behavior both in temporal order and in relation to each one of the independent variables. However, the introduction of new independent variables may improve the obtained function. Hence, new independent terms have been included as an attempt to improve the model, thus creating a new equation. As may be noted the, Eq. (10) is non linear in the variables $X^{2}, Y^{2}, Z^{2}, X Y, X Z$ and $Y Z$. Thus, it is important to make a transformation in the independent variables with the objective of simplifying the calculation of the coefficients of regression.

$$
E_{X i}=\beta_{X 0}+\beta_{X 1} X_{i}+\beta_{X 2} Y_{i}+\beta_{X 3} Z_{i}+\beta_{X 4} X_{i}^{2}+\beta_{X 5} Y_{i}^{2}+\beta_{X 6} Z_{i}^{2}+\beta_{X 7} X_{i} Y_{i}+\beta_{X 8} X_{i} Z_{i}+\beta_{X 9} Y_{i} Z_{i}+\varepsilon_{X i}(10)
$$

In this case, it is recommended the substitution of the variables as follows: $X=Z_{1} ; \quad Y=Z_{2} ; \quad Z=Z_{3} ; \quad X^{2}=Z_{4} ; \quad Y^{2}=Z_{5} ; Z^{2}=Z_{6} ; X Y=Z_{7}$; $X Z=Z_{8} ; Y Z=Z_{9}$. As a result of this transformation Eq. (11), which is a multiple linear regression equation, is obtained.

$E_{X i}=\beta_{X 0}+\beta_{X 1} Z_{1 i}+\beta_{X 2} Z_{2 i}+\beta_{X 3} Z_{3 i}+\beta_{X 4} Z_{4 i}+\beta_{X 5} Z_{5 i}+\beta_{X 6} Z_{6 i}+\beta_{X 7} X_{7 i}+\beta_{X 8} Z_{8 i}+\beta_{X 9} Z_{9 i}+\varepsilon_{X i}(11)$

Before realizing the calculation of the coefficients, all their coefficients have been tested. Only the coefficients of the independent variable that are highly correlated to the answer or dependent variables were calculated. The procedure used to select the significant variables in the regression was the one called "stepwise" (Draper \& Smith, 1981). This way the mathematical equations that describe input-output of the system "measuring machine" in the preferential directions $X, Y$ and $Z$ respectively were obtained (Eq. 12).

$E x=0,0875 \cdot Z-0,03428 \cdot Y+0,00003 \cdot X^{2}+0,00006 \cdot Z^{2}+0,00031 \cdot Y^{2}-0,00015 \cdot X Z$

$E y=0,08204 X-0,70775 Z+0,00011 Y^{2}+0,00011 Z^{2}-0,00005 Y X$

$E z=0,10429 \cdot Z-0,06733 \cdot X+0,01186 \cdot Y+0,00015 \cdot Z^{2}-0,00008 \cdot Y^{2}-0,00002 \cdot Z X$

With the objective of ensuring that in the point $X=Y=Z=0$ the volumetric error is zero the regression was moved to the origin. As a consequence, the values of the coefficients $\beta_{0}$ in the regression equations that described the components of the volumetric error are equal to zero.

\section{Evaluation of the Proposed Model}

The proposed model was statistically and experimentally evaluated. The statistic evaluation covers the calculation of the correlation coefficients of the sample, an analysis of the residues and the verification of the hypotheses. The experimental evaluation was carried out through the comparison of the results predicted by the model with the results obtained from the measurement of a bal bar in some positions and orientations in the working volume of the machine as requested by the standard ANSI/ASME B89.4.1 (1995).

\section{Statistic Evaluation of the Model}

In the statistic evaluation of the model the first step consists in the calculation of the correlation coefficients of the sample $\left(r^{2}\right)$ for each obtained equation. The calculated correlation coefficients were of $99.19,99.93$ and $98.15 \%$ for the equations that described components of the volumetric error in the direction of the axes $X, Y$ and $Z$, respectively. This means that a good percentage of variability of each one of the components of the volumetric error is explained by the obtained regression equations, which indicates that the proposed model is adequate to describe the analyzed data.

The statistic evaluation to the residue began with the analysis of the values of the graph of the predicted components of volumetric error in function to the regression residues (Fig. 4).

The values of the residue of $E x$ equation are randomly distributed around zero for all cases. That shows that the specification is appropriate. It is also possible to observe that the residues are in the interval $\pm 4 \mu \mathrm{m}$, approximately.

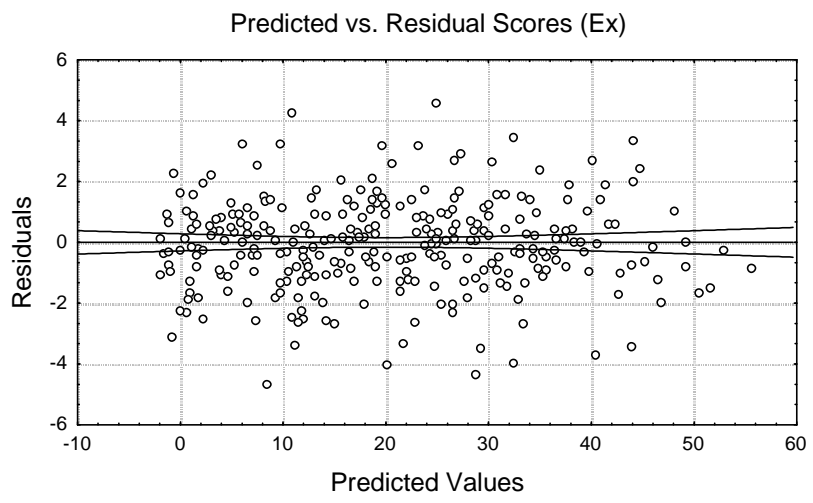

Figure 4. Regression residues graph in function of expected values for Ex equation.

It has been observed a good behavior of the residues according to time, i.e., they are randomly distributed around zero with no bias. This shows that the experiments have been properly carried out, i.e., the components of the volumetric error have been correctly established. These results indicated that incorrect instrumentation manipulation and great variability in the conditions of test have not occurred.

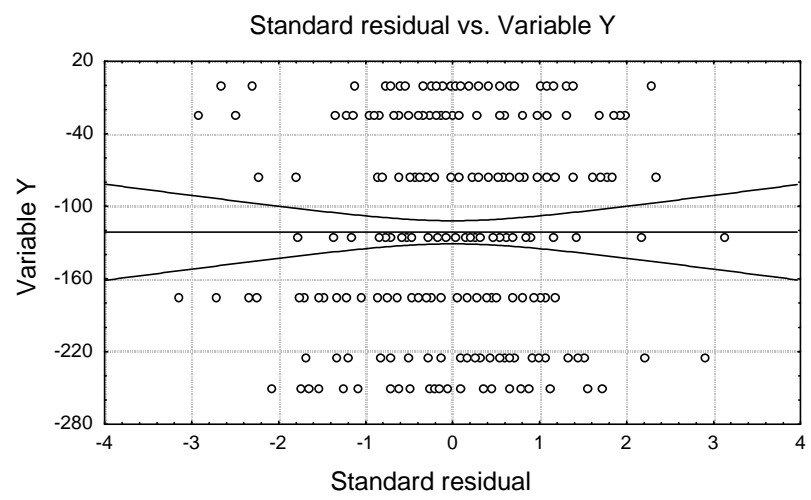

Figure 5. Residues of the regression equation for component Ex versus variable $\mathrm{Y}$.

It is observed in the graph several levels defined by constant values of the variables $X, Y$ and $Z$. These values represent each one of the generators of the measure. In the specific case of the component of the volumetric error $E x$ it is obtained 13, 7 and 7 levels in the variables $X, Y$ and $Z$, respectively. The variable $X$ takes values in the interval 0 to $300 \mathrm{~mm}$ with spacing of $35 \mathrm{~mm}$. For the variable $Y$ these values vary between 50 to $350 \mathrm{~mm}$ with spacing of $50 \mathrm{~mm}$, whereas the values of the variable $Z$ are between -35 and 235 with spacing of $50 \mathrm{~mm}$ (still the values $-10 \mathrm{~mm}$ and $-260 \mathrm{~mm}$ ). 
In Fig. 5 it is possible to observe that the residues are randomly distributed around zero for the independent variable. Similar results have been observed for all the other variables. This confirms that the specification is appropriate. In the same way it has been constructed the graphs of the residue in function to the independent variables for the regression equations of the components Ey and $E z$. The results were alike and therefore they are not here presented.

Besides the analysis previously presented, normality test for the residues using the normal probability plots have been done. The result of this test for the equation of $E x$ is presented in Fig. 6. It is possible to observe that almost all the values of the residue are on a theoretical straight line. This way, the hypothesis of the normality of distribution cannot be rejected. Similar results were observed in the analysis of residue graphs of the regression equations of $E y$ and $E z$.



Figure 6. Normal probability plot of residues.

It has also been constructed histograms for each one of the groups of residues, confirming that they presented a nearly normal distribution, with an average almost like zero for all cases and standard deviation of $0.99 ; 1.73$ and $0.76 \mu \mathrm{m}$, respectively. The confidence interval used was of $95 \%$.

In conclusion, the residues of the regression equations which describe the components of volumetric error, generated in the work volume of the machine in the preferential directions of movement, presented normal distribution of probabilities. According to what was previously shown, the regression equations obtained are considered adequate to describe the behavior of the components of volumetric error of the Three Coordinate Measuring Machine analyzed.

\section{Verification of the Proposed Model Through Measurement of a Calibrated Ball Bar}

Another verification of the proposed model was done through comparison of the results predicted by the model and the ones obtained through the measurement of a ball bar in different positions taken from suggestion of the standard ANSI/ASME B 89.4.1 (1995). This standard recommends the measurement of a noncalibrated ball bar in 20 different positions and orientations in the working volume of the machine. It has been devised to use a ball bar with known nominal length to raise the necessary data. For time saving reasons, the measurement was carried out in 12 of the 20 positions recommended by the standard. The positions of the bar were chosen in order for it to be parallel to the direction of the axes $X, Y$ and $Z$, in the diagonal of the plans $X Y, X Z$ and $Y Z$ and in the volumetric diagonals.

The measurement of the ball bar consists in the determination of the distance between the centers of the balls in the bar. For that it was necessary to measure the diameter of the balls, to calculate the coordinates of their centers and the distance between them. The distance between the centers of the bar is conventionally called the length of the bar. For distinct positions, the balls were measured five times and the coordinates $X, Y$ and $Z$ of the measuring points were collected. It is an indispensable requisite that these points are linearly independent.

From the collected coordinate points and the ball equation, the least squares method was applied. The coordinates of the centers of the balls and their respective averages in each position were calculated. After that, the length of the bar was calculated $\left(D_{B M}\right)$. It is known that for distinct points belonging to the working volume of the machine the volumetric errors may present different directions among them and different measuring directions of the bar. Therefore, before calculating the real length of the bar, the volumetric errors must be projected in a direction of measurement, i.e., in the direction of the bar. The real length of the bar $\left(D_{B R}\right)$ is calculated as the difference between the measured length $\left(D_{B M}\right)$ and the projection of the volumetric error $\left(P_{E B}\right)$ in the direction of the bar.

$$
D_{B R}=D_{B M}-P_{E B}
$$

For that, the numerical values of the components of the volumetric error were synthesized using the obtained regression equations. After, the centers of the balls and their respective volumetric errors were calculated. Once these values have been acquired they are projected in the direction of measurement. In order to do that, the director co-sine which define the orientation of the bar inside the working volume of the machine were calculated.

The projections of the bar in the preferential directions are determined from the coordinates of the centers of the balls 1 and 2 .

$$
\begin{aligned}
& D_{B M x}=X_{2}-X_{1} \\
& D_{B M y}=Y_{2}-Y_{1} \\
& D_{B M z}=Z_{2}-Z_{1}
\end{aligned}
$$

The angles that define the position of the bar with the directions $O X, O Y$ and $O Z$ are designated by $\alpha, \beta$ and $\gamma$. Therefore, the director co-sines may be calculated as:

$$
\begin{aligned}
& \cos \alpha=D_{B M x} / D_{B M} \\
& \cos \beta=D_{B M y} / D_{B M} \\
& \cos \gamma=D_{B M z} / D_{B M}
\end{aligned}
$$

So, the projections of the components of the volumetric error $E x, E y$ and $E z$ are calculated in the direction of the bar, denoted by $E_{B X}, E_{B Y}$ and $E_{B Z}$, using the Eq. (16).

$$
\begin{aligned}
& E_{B x}=E_{X} \cos \alpha \\
& E_{B y}=E_{Y} \cos \beta \\
& E_{B z}=E_{Z} \cos \gamma
\end{aligned}
$$

Therefore, the projection of the volumetric error in the direction of the bar $\left(E_{B N}\right)$ for the center points of the balls 1 and 2, respectively, is given by Eq. (17).

$$
\begin{aligned}
& E_{B 1}=E_{B_{x} 1}+E_{B y 1}+E_{B z 1} \\
& E_{B 2}=E_{B_{x} 2}+E_{B y 2}+E_{B z 2}
\end{aligned}
$$

Whereas the measurement error is the difference in the projections of the volumetric errors.

$$
P_{E B}=E_{B 1}-E_{B 2}
$$


Eventually, the value of the calculated volumetric error $\left(P_{E B}\right)$ is corrected from the measured length of the bar $\left(D_{B M}\right)$, thus obtaining the real length $\left(D_{B R}\right)$.

$$
D_{B R}=D_{B M}-P_{E B}
$$

In order to evaluate the effectiveness of the proposed model for the prediction of volumetric error it is necessary to determine the difference between the calculated length values and the standard length $\left(D_{B P}\right)$. The standard length of the ball bar may be determined through a calibration procedure. In order to do that, it was used a universal measuring machine made by Societe Geneovoise D' Instruments de Physique (SIP), model $302 \mathrm{M}$, whose resolution and uncertainty are $0.1 \mu \mathrm{m}$ and $\pm 0.1 \mu \mathrm{m}$, respectively. Both the distance between the extremities of the balls and their diameters were measured nine times, since for this number of measurements, the standard deviation value becomes stable. The values of the averages and standard deviations were calculated and used for the determination of the standard length of the ball bar. As a result it has been found that the standard length or dimension of the bar is of $197.486 \pm 0.001 \mathrm{~mm}$, with $99.7 \%$ confidence. Once the real length and the standard length of the bar have been obtained, it is possible to determine the difference between these values through Eq. (19). This difference is denominated residual error.

$$
\text { Re sidual error }=D_{B R}-D_{B P}
$$

From the analyzes of Fig. 7 it is found that in the positions 1,2, 3,4 and 6 corresponding to diagonals in plans $X Y, X Z$ and $Y Z$, the values of the residual error are in the interval $\pm 5 \mu \mathrm{m}$. These results may be considered adequate. The positions 8,9 and 10 corresponding to volumetric diagonals presented values of residual errors of up to $7 \mu \mathrm{m}$ in the position 8 . These values are higher than the expected ones. In the positions $11,16,17$ and 18 corresponding to preferential directions present adequate values of residual errors, except for the position 17, whose residual error is of $9 \mu \mathrm{m}$.



Figure 7. Comparison results with the method of standard ANSI/ASME B89.4.1 (1995).

The fact that the volumetric error in the direction of the axis $X$ may reach values of up to $240 \mu \mathrm{m}$, the value of $9 \mu \mathrm{m}$ in the position 17 may be considerer good, because it represents $3.75 \%$ of the maximum error in that direction. The same occurs with the value of $7 \mu \mathrm{m}$ in the position 8 , which correspond to one of the diagonals of the volume with positive moving direction for the axis $X$ and negative for the axis $Y$ and $Z$. The fact that values of residual errors outside the interval $\pm 6 \mu \mathrm{m}$ are obtained may be explained by the absence of the probing system effect. During the collection of data in order to obtain the proposed model, the error was found using the interferometric system. It is known that during the measurement of the ball bar the probing system effect is included.
A normality test was applied to the numerical values of the residual error and shown in a graph of normal probability. The normality test has shown values of residual error with small deviations in relation to the theoretical straight line, however, the hypothesis of normality of the residues may be accepted. It has also been calculated some statistics in order to characterize this distribution such as the average, the standard deviation and the coefficients kurtosis and skewness, whose values are of respectively:-0.94, $4.12,0.75$ and $0.01 \mu \mathrm{m}$.

\section{Estimation of Uncertainty Associated of Volumetric Error Components}

Finally, estimation of the measurement uncertainty associated to the components of volumetric error was performed. In order to accomplish the task, the law of uncertainty propagation was applied to equations that described these components, according to the Guide to the expression of uncertainty in measurement (ISO TAG, 1993).

Eq. (21) allows the determination of the values of the component of volumetric error, $E x$.

$$
E_{x}=\text { Positioning error }+ \text { correction Factor }
$$

The Eq. (22) allows the determination of the uncertainty associated to the positioning errors measurement. This equation is based on the fact of that the values of the components of volumetric error was defined as being the difference between the reading value of the machine and the value indicated by the laser. One can still incorporate to the model all the influence variables and the correction factors (Valdés, et al. 2005).

$$
E_{\text {Pos }}=M+R_{C M M}+R_{L}+\text { Thermal effect }
$$

According to ISO/TR 16015 (2003) the uncertainty associated to the measurements of lengths due to thermal effects must consider the uncertainty associated to differential expansion between the measurand and the standard, the uncertainty associated to the measurement of temperature and the uncertainty associated to the variation of room temperature compared to the reference temperature.

Adding the terms related the thermal effects and applying the law of propagation of uncertainties in Eq. (22), one can rewrite it as Eq. (23), which allows estimating the uncertainty associated to the positioning error.

$$
\begin{gathered}
u^{2}\left(E_{P o s}\right)=\left(\frac{\partial E_{P o s}}{\partial M}\right)^{2}\left(u_{e}\right)^{2}+\left(\frac{\partial E_{P o s}}{\partial R_{C M M}}\right)^{2}\left(u_{R_{C M M}}\right)^{2}+\left(\frac{\partial E_{P O S}}{\partial R_{L}}\right)^{2}\left(u_{R_{l r}}\right)^{2}+ \\
+\left(\frac{\partial E_{P_{O S}}}{\partial \Delta T_{L}}\right)^{2}\left(u_{\Delta T_{L}}\right)^{2}+\left(\frac{\partial E_{P O S}}{\partial \Delta T_{E}}\right)^{2}\left(u_{\Delta T_{E}}\right)^{2}+\left(\frac{\partial E_{P_{O S}}}{\partial \alpha_{L}}\right)^{2}\left(u_{\alpha_{L}}\right)^{2}+ \\
+\left(\frac{\partial E_{P o s}}{\partial \alpha_{E}}\right)^{2}\left(u_{\alpha_{E}}\right)^{2}
\end{gathered}
$$

where: EPos is the positioning error; $M$ is the value indicated by machine; $P$ is the reference value (Laser); $\alpha_{E}$ is the coefficient of thermal expansion of the scale (glass); $\alpha_{P}$ is the coefficient of thermal expansion of the laser beam; $\Delta T_{P}$ is the difference between the room temperature and the reference temperature; $\Delta T_{E}$ is the difference between the scale temperature and the reference temperature; $R_{\text {Laser }}$ is the resolution of the laser and $R_{C M M}$ is the resolution of the machine.

Table 1 presents the data regarding the calculation of the uncertainty of the $X$-axis positioning errors. 
Table 1. Positioning error measurement uncertainty analysis PosX100.

\begin{tabular}{l|l|l|l|l|l}
\hline $\begin{array}{l}\text { Source of } \\
\text { uncertainty }\end{array}$ & $\begin{array}{l}\text { Uncertainty } \\
\text { type }\end{array}$ & $\begin{array}{l}\text { Probability } \\
\text { distribution }\end{array}$ & $\begin{array}{l}\text { Sensitivity } \\
\text { coefficient }\end{array}$ & $\begin{array}{l}\text { Degrees of } \\
\text { freedom. }\end{array}$ & $\begin{array}{l}\text { Standard } \\
\text { uncertainty }\end{array}$ \\
\hline $\mathrm{M}$ & $\mathrm{A}$ & Normal & $1 \mu \mathrm{m}$ & 4 & 0.487 \\
\hline $\mathrm{R}_{\mathrm{MM} 3 \mathrm{c}}$ & $\mathrm{B}$ & Rectangular & $1 \mu \mathrm{m}$ & $\infty$ & $1.15 \mathrm{E}-6$ \\
\hline $\mathrm{R}_{\text {laser }}$ & $\mathrm{B}$ & Rectangular & $1 \mu \mathrm{m}$ & $\infty$ & 0.0058 \\
\hline$\alpha_{\mathrm{E}}$ & $\mathrm{B}$ & Rectangular & $0.012 \mu \mathrm{m}^{0} \mathrm{C}$ & $\infty$ & $1.11 \mathrm{E}-29$ \\
\hline $\mathrm{C}_{\text {laser }}$ & $\mathrm{B}$ & Rectangular & $0.012 \mu \mathrm{m}^{0} \mathrm{C}$ & $\infty$ & $7.76 \mathrm{E}-23$ \\
\hline$\delta \mathrm{T}$ & $\mathrm{B}$ & Rectangular & $-8.34 \mathrm{E}-24 \mu \mathrm{m} /{ }^{\circ} \mathrm{C}$ & $\infty$ & $-1.07 \mathrm{E}-8$ \\
\hline$\Delta \mathrm{T}$ & $\mathrm{B}$ & Rectangular & $1.69 \mathrm{E}-21 \mu \mathrm{m} /{ }^{\circ} \mathrm{C}$ & $\infty$ & $6,67 \mathrm{E}-23$ \\
\hline Combined standard uncertainty $\left(u_{c}\right)$ in $\mu \mathrm{m}$ & & 0.487 \\
\hline Effective degrees of freedom $\left(v_{\text {eff }}\right)$ & & 4.00 \\
\hline Coverage factor $\left(v_{\text {eff }}, 95 \%\right)$ & $\mathrm{k}=2.78$ \\
\hline Expanded uncertainty $(95 \%)$ in $\mu \mathrm{m}$ & 1.354
\end{tabular}

Combined standard uncertainty of positioning error in position $X=100 \mathrm{~mm}$ was $0.487 \mu \mathrm{m}$, whereas expanded uncertainty was $1.354 \mu \mathrm{m}$. Uncertainty values for all positions in the working volume of the machine vary between 0.27 e $0.58 \mu \mathrm{m}$. The presence of differences can be attributed to the variability of results. Similar results of the uncertainty associated to the positioning errors were obtained for all axes and for any position.

The correction factor was obtained by adding two displacements using the mechanical square standard and LVDT type transducer.

Eq. (24) allows the estimating of the uncertainty associated to the displacement $D_{l}$.

$$
D_{1}=L_{L V D T}+C_{S q}+R_{L V D T}+L \cdot \alpha_{S q} \cdot \Delta T_{E}
$$

where: $D_{l}$ is the measured displacement; $\mathrm{L}_{\mathrm{LVDT}}$ is the reading taken by LVDT; $C_{S q}$ is the correction due to error of the mechanical square; $R_{L V D T}$ is the resolution of the LVDT; $\alpha_{S q}$ is the coefficient of thermal expansion of the mechanical square (granite) and $\Delta T_{E}$ is the difference between the mechanical square temperature and the reference temperature.

By applying the law of propagation of uncertainties in the Eq. (24), one can write:

$$
\begin{gathered}
u^{2}\left(D_{1}\right)=\left(\frac{\partial D_{1}}{\partial L_{L V D T}}\right)^{2}\left(u_{L_{L V D T}}\right)^{2}+\left(\frac{\partial D_{1}}{\partial C_{S_{q}}}\right)^{2}\left(u_{C_{S q}}\right)^{2}+\left(\frac{\partial D_{1}}{\partial R_{L V D T}}\right)^{2}\left(u_{R_{L V D T}}\right)^{2}+ \\
+\left(\frac{\partial D_{1}}{\partial \alpha_{S q}}\right)^{2}\left(u_{\alpha_{S q}}\right)^{2}+\left(\frac{\partial D_{1}}{\partial \Delta T_{S q}}\right)^{2}\left(u_{\Delta S_{S q}}\right)^{2}
\end{gathered}
$$

Table 2 presents the data regarding the calculation of the uncertainty of the displacement $D_{l}$.

Table 2. Displacement, D1, measurement uncertainty analysis.

\begin{tabular}{l|l|l|l|l|l}
\hline $\begin{array}{l}\text { Source of } \\
\text { uncertainty }\end{array}$ & $\begin{array}{l}\text { Uncertainty } \\
\text { type }\end{array}$ & $\begin{array}{l}\text { Probability } \\
\text { distribution }\end{array}$ & $\begin{array}{l}\text { Sensitivity } \\
\text { coefficient }\end{array}$ & $\begin{array}{l}\text { Degrees of } \\
\text { freedom. }\end{array}$ & $\begin{array}{l}\text { Standard } \\
\text { uncertainty }\end{array}$ \\
\hline $\mathrm{L}_{\mathrm{LVDT}}$ & $\mathrm{A}$ & Normal & 1 & 10 & $0.05 \mu \mathrm{m}$ \\
\hline $\mathrm{C}_{\mathrm{Sq}}$ & $\mathrm{B}$ & Rectangular & 1 & $\infty$ & $6.99^{*} \mathrm{E}-6 \mu \mathrm{m}$ \\
\hline $\mathrm{R}_{\mathrm{LVDT}}$ & $\mathrm{B}$ & Rectangular & 1 & $\infty$ & $4.08^{*} \mathrm{E}-5 \mu \mathrm{m}$ \\
\hline \multicolumn{5}{l}{ Combined standard uncertainty $\left(u_{c}\right)$ in $\mu \mathrm{m}$} & 0.05 \\
\hline \multicolumn{2}{l}{ Effective degrees of freedom $\left(v_{\text {eff }}\right)$} & 4.00 \\
\hline \multicolumn{2}{l}{ Coverage factor $\left(v_{\text {eff }}, 95 \%\right)$} & $\mathrm{k}=2.78$ \\
\hline
\end{tabular}

Combined standard uncertainty of orthogonal error was $0.05 \mu \mathrm{m}$, whereas expanded uncertainty was $0.139 \mu \mathrm{m}$. The results obtained at the different positions vary between 0.050 and $0.467 \mu \mathrm{m}$. The observed differences can be credited to the operator who is in charge of the carriage movement, because the evaluated machine is manual. If the operator is not well trained and extremely careful, he/she may produce strengths in the direction of the measured displacement, and this may consequently alter the measurement results.

Next, uncertainties associated to the components of the volumetric error $E x, E y$ and $E z$ were calculated. Uncertainty values indicate that at any point in the working volume of the $3 \mathrm{CMM}$, the volumetric error components present standard expanded uncertainty values close to $0.525,0.447$ and $0.727 \mu \mathrm{m}$, respectively. These results may be considered adequate and that may be attributed to the measurement of the volumetric error components using the space grid method.

\section{Conclusions}

The main results obtained in the development of this work may be summarized and presented as the conclusions below.

The regression equations which describe the components of the volumetric error in the directions $X, Y$ and $Z$ present relation coefficients of $99.19,99.93$ and $98,15 \%$, respectively.

The numerical values of the residue for each one of the equations of the proposed model are of $\pm 4, \pm 4$ and $\pm 2 \mu \mathrm{m}$, for each one of the preferential directions $X, Y$ and $Z$, respectively. Therefore, in case a new compensation system is implemented from this model, no matter the position of measurement, the volumetric error made after the compensation will not be over $\pm 6 \mu \mathrm{m}$.

The variable $Z$, which in this case represents the coordinate $Z$ of the measured point, is the one that influences the most the numerical values of the volumetric error in the three directions. The proposed method may be extended to all Three Coordinate Measuring 
Machines, although its application is more adequate for the $3 \mathrm{CMM}$ with higher degree of automation. Therefore, the experimentation time required for the use of the space grid method may be considerably reduced.

When compared to the synthesizing method, the proposed model is mathematically simpler and it does not need the calibration of the twenty one geometric errors.

The procedures described in ISO TAG (1993) have been efficient to determine the uncertainty associated the components of the volumetric error collected through a direct calibration procedure (space grid method).

The standard expanded uncertainty values associated to volumetric error components $E x, E y$ and $E z$ were $0.525,0.447$, and $0.727 \mu \mathrm{m}$, respectively. These results may be considered small and that may be attributed to the measurement of the volumetric error components using the space grid method.

\section{Acknowledgements}

The authors are grateful to Conselho Nacional de Desenvolvimento Científico e Tecnológico - $\mathrm{CNPq}$ and the Fundação de Amparo à Pesquisa do Estado de São Paulo - FAPESP (Process number 03/00709-8) for supporting this work.

\section{References}

ANSI/ASME B 89.4.1., 1995, "Methods for performance evaluation of Coordinate Measuring Machines”.

Box, G.E.P.; Hunter, W.G. \& Hunter, J.S. 1978. "Statistic for experimenters - an introducing to design, data analysis, and model building. John Wiley \& Sons. New York. p. 1-653.
Burdekin, M.S., Di Giacomo, B. Xijing, Z., 1984. "Calibration software and application to Coordinate Measuring Machines". Departament of Mechanical Engineering. UMIST, Manchester, UK, pp 01-07.

Draper, N.R., Smith, H., 1981, "Applied regression analysis", In charter 10: An introduction to Nonlinear Estimation, pp. 458-529.

ISO TAG 4/WG 3, 1993, "Guide to the expression of uncertainty in measurement", Geneva Switzerland.

ISO/TR 16015, 2003, "Geometrical product specifications (GPS) Systematic errors and contributions to measurement uncertainty of length measurement due to thermal influences". Technical report.

Harris, C.M., 1996, "Shock and vibration Handbook", Quarta edição, McGRAW-Hill, New York, pp. 21.8; 21.21; 22.9; 23.9; 27.8 .

Hoffmann, R. \& Vieira, S. 1973. "Análise de regressão" (In Portuguese). Editora HUCITEC, São Paulo. SP. Brazil.

Kunzmann, H. \& Waldele, F., 1988, "Performance of CMMs", Annals of the CIRP, Vol. 39, No. 2, pp. 633-640.

Ogata, K., 1982, "Engenharia de controle moderno" (In Portuguese). Capítulo 4, Modelos matemáticos de sistemas físicos.Editora Prentice Hall do Brazil, Rio de Janeiro. Brazil.

Martinez Orrego, R.M. 1999. "Método de calibração direta para Máquinas de Medir a Três Coordenadas" (In Portuguese). PhD Thesis Escola de Engenharia de São Carlos, Universidade de São Paulo, São Carlos, SP. Brazil. 175p.

Spiegel, M.R. (1974). "Estatística". Capítulo 13. Ajustamento de curvas e o método dos mínimos quadrados. (In Portuguese). Editora McGraw Hilldo Brasil, Ltda. pp. 362-400.

Valdés, R.A., 1999, "Equacionamento das componentes de erro volumétrico em Máquinas de Medir a Três Coordenadas" (In Portuguese). MSc Dissertation - Escola de Engenharia de São Carlos, Universidade de São Paulo, São Carlos, SP. Brazil. 181p.

Valdés, R.A., Di Giacomo, B., Sato D.P.V., 2005, "Cálculo da incerteza de medição associada aos erros geométricos em uma MM3C" (In Portuguese). $3^{0}$ COBEF. 12 a 15 de Abril - Joinville. SC Brazil, registered in CD. 